\title{
Editorial
}

\section{Special Issue on Critical Assessment of Theoretical Calculations of Atomic Structure and Transition Probabilities}

\section{Per Jönsson ${ }^{1}$ and Hyun-Kyung Chung ${ }^{2, *}$}

1 School of Technology, Applied Mathematics Group, Malmö University, Malmö, Sweden; E-Mail: per.jonsson@mah.se

2 International Atomic Energy Agency, Atomic and Molecular Data Unit, Nuclear Data Section, P.O. Box 100, A-1400 Vienna, Austria

* Author to whom correspondence should be addressed; E-Mail: h.chung @iaea.org;

Tel.: +43-1-2600 21729.

Received: 19 June 2013 / Accepted: 20 June 2013 / Published: 21 June 2013

There exist several codes in the atomic physics community to generate atomic structure and transition probabilities freely and readily distributed to researchers outside atomic physics community, in plasma, astrophysical or nuclear physics communities. Users take these atomic physics codes to generate the necessary atomic data or modify the codes for their own applications. However, there has been very little effort to validate and verify the data sets generated by non-expert users.

In a recent IAEA meeting, researchers who develop the atomic physics codes met to discuss procedures to validate data sets generated by these distributed atomic physics codes. They agreed to implement and document the procedures to insure and validate code-generated data for non-experts in their codes.

This special issue aims to document each code's approach and procedure to critically assess the uncertainties of theoretical atomic data will have a broad impact, not only for the atomic physics community, but also for other communities interested in high quality atomic data.

(C) 2013 by the authors; licensee MDPI, Basel, Switzerland. This article is an open access article distributed under the terms and conditions of the Creative Commons Attribution license (http://creativecommons.org/licenses/by/3.0/). 